Portland State University

PDXScholar

$9-1-2006$

\title{
Relativistic corrections to a generalized sum rule
}

P.T. Leung

Portland State University

H. Sinky

Follow this and additional works at: https://pdxscholar.library.pdx.edu/phy_fac

Part of the Physics Commons

Let us know how access to this document benefits you.

\section{Citation Details}

Sinky, H. H., \& Leung, P. T. (2006). Relativistic corrections to a generalized sum rule. Physical Review A (Atomic, Molecular, And Optical Physics), 74(3), 34703-1-4.

This Article is brought to you for free and open access. It has been accepted for inclusion in Physics Faculty Publications and Presentations by an authorized administrator of PDXScholar. Please contact us if we can make this document more accessible: pdxscholar@pdx.edu. 


\title{
Relativistic corrections to a generalized sum rule
}

\author{
H. Sinky and P. T. Leung* \\ Department of Physics, Portland State University, P.O. Box 751 Portland, Oregon 97207, USA
}

(Received 31 December 2005; revised manuscript received 20 July 2006; published 12 September 2006)

\begin{abstract}
Relativistic corrections to a previously established generalized sum rule are obtained using the FoldyWouthysen transformation. This sum rule derived previously by Wang [Phys. Rev. A 60, 262 (1999)] for a nonrelativistic system contains both the well-known Thomas-Reiche-Kuhn and Bethe sum rules, for which relativistic corrections have been obtained in the literature. Our results for the generalized formula will be applied to recover several results obtained previously in the literature, as well as to another sum rule whose relativistic corrections will be obtained.
\end{abstract}

DOI: 10.1103/PhysRevA.74.034703

It is well known that in spite of the complications in the calculation of many quantum processes, the application of sum rules leads to great simplifications in many calculations such as those for the energy loss of charged particles. Examples can be found in almost all quantum systems including subatomic, atomic, and condensed matter systems [1]. However, in most of these applications, the system must be strictly nonrelativistic with its eigenstates satisfying the Schrödinger equation. Some famous examples are the Thomas-Reiche-Kuhn (TRK) and the Bethe sum rule applied to nonrelativistic atomic systems. This is so restricted since the derivation of these sum rules is based on the completeness of the eigenstates, and a relativistic system will have to include the negative energy states [2] which will either lead to trivial sums or uninteresting results of little applications.

The problem of relativistic corrections to various sum rules has a long history, and there have been different approaches to suppress the negative energy states in doing the sum [3]. These include the projection operator method [4], the second-quantization method [5], and the semirelativistic approach $[3,6]$. For systems such as heavy atoms, these relativistic corrections will be significant and will quantitatively modify various physical quantities calculated using the nonrelativistic sum rules. For example, we had previously used the semirelativistic approach to obtain relativistic corrections to the Bethe sum rule [3,7], and the results were later applied to obtain significant corrections to the Bethe stopping power theory [8]. While the Bethe sum rule and its "dipolar limit" (i.e., the TRK sum rule) are both very useful in atomic physics, they have been generalized by Wang [9] recently for a nonrelativistic system. In this Brief Report, we shall extend our previous work based on the Foldy-Wouthuysen (FW) transformation $[3,7]$ to obtain relativistic corrections to the results of Wang [9] and recover the previous corrections in the case of the TRK and the Bethe sum rules under appropriate conditions. We first start by reformulating Wang's result following an approach that allows the sum to be calculated for an operator (generally non-Hermitian) which is an arbitrary function of the spatial coordinates.

For an arbitrary function of the coordinate operator $F(\vec{r})$, Wang [9] obtained the following sum rule for a nonrelativistic system with $H=p^{2} / 2 m+V(\vec{r})$ :

\footnotetext{
${ }^{*}$ Corresponding author.
}

PACS number(s): 03.65.Nk, 32.70.Cs

$$
\sum_{n}\left(E_{n}-E_{0}\right)|\langle n|F(\vec{r})| 0\rangle|^{2}=\frac{\hbar^{2}}{2 m}\left\langle 0\left|\vec{\nabla} F \cdot \vec{\nabla} F^{\dagger}\right| 0\right\rangle,
$$

and a similar result when $F$ itself is a vector function. Care must be taken to allow $F$ in Eq. (1) to be non-Hermitian in general [10]. In this case, one should have instead the following more general result $[1]$ :

$$
\begin{aligned}
\sum_{n}\left(E_{n}-E_{0}\right)|\langle n|F(\vec{r})| 0\rangle|^{2} \\
=\left\langle 0\left|F^{\dagger}[H, F]\right| 0\right\rangle \\
=\frac{1}{2}\left\langle 0\left|F^{\dagger}[H, F]+\left[F^{\dagger}, H\right] F\right| 0\right\rangle \\
=\frac{\hbar^{2}}{4 m}\langle 0|\left(F \nabla^{2} F^{\dagger}-F^{\dagger} \nabla^{2} F\right) \\
+2\left(F \vec{\nabla} F^{\dagger}-F^{\dagger} \vec{\nabla} F\right) \cdot \vec{\nabla}+2 \vec{\nabla} F \cdot \vec{\nabla} F^{\dagger}|0\rangle,
\end{aligned}
$$

which reduces to the result in Eq. (1) for Hermitian $F$. As an example, for $\vec{F} \equiv \vec{r}$ which is Hermitian, both Eqs. (1) and (2) reduce back to the well-known nonrelativistic TRK sum rule [11]. As another example, let $F=e^{i \vec{q} \cdot \vec{r}}$ where $\vec{q}$ is the momentum transfer; one easily shows from Eq. (2c) the following result [11]:

$$
\sum_{n}\left(E_{n}-E_{0}\right)\left|\left\langle n\left|e^{i \vec{q} \cdot \vec{r}}\right| 0\right\rangle\right|^{2}=\frac{\hbar^{2}}{2 m}\left\langle 0\left|q^{2}-2 i \vec{q} \cdot \vec{\nabla}\right| 0\right\rangle .
$$

Since the expectation value of the momentum operator $\langle n|\vec{p}| n\rangle=0$ with respect to any eigenstate of the nonrelativistic Hamiltonian, the second term on the right-hand side (RHS) of Eq. (3) vanishes and one sees immediately that Eq. (3) yields back the well-known Bethe sum rule $[11,12]$. In the following, we shall calculate the lowest-order relativistic corrections to the result in (2) for a single-particle system and see how the previous results obtained in the literature for the relativistic corrections to the TRK and Bethe sum rules [3] can be recovered.

To obtain to lowest order the relativistic corrections to Eq. (2), we follow our previous approach [3] for a one-electron system using the FW transformation. By adopting the independent-particle, local-potential description for a manyelectron atom, this one-particle result can be utilized to obtain first approximations to the corrections for a manyelectron system $[8,13]$. Hence we start with our semirelatistic Hamiltonian in the form [3] 


$$
\begin{aligned}
\hat{H}= & m c^{2}+\frac{p^{2}}{2 m}-\frac{p^{4}}{8 m^{3} c^{2}}+e V(\vec{r})-\frac{\hbar e}{4 m^{2} c^{2}} \vec{\sigma} \cdot(\vec{E} \times \vec{p}) \\
& -\frac{\hbar^{2} e}{8 m^{2} c^{2}} \vec{\nabla} \cdot \vec{E}
\end{aligned}
$$

where we have included the rest mass, the kinetic energy correction, the spin-orbit, and the Darwin terms in the Schrödinger equation for the one-electron system and $V=$ $-Z e / r$ is the Coulomb potential due to the nuclear charge. It has been pointed out previously $[3,5]$ that in such a calculation of the sum rule in (2), the FW transformation must be applied not only to the Hamiltonian, but also to the operator function $F(\vec{r})$. When this is done, we obtain the "transformed $F(\vec{r})$ " to have the following form [14]:

$\hat{F}(\vec{r})=F(\vec{r})+\frac{\hbar^{2}}{8 m^{2} c^{2}} \vec{\nabla} \cdot \vec{G}+\frac{\hbar}{4 m^{2} c^{2}} \vec{\sigma} \cdot \vec{G} \times \vec{p}+\frac{\hbar^{2} e}{4 m^{3} c^{4}}(\vec{E} \cdot \vec{G})$,

where $\vec{G} \equiv \vec{\nabla} F$. In the following, we shall use the $\hat{H}$ in Eq. (4) and $\hat{F}$ in Eq. (5) into Eq. (2a) to compute the relativistic corrections to (2). To the lowest order-i.e., $O\left(\mathrm{~m}^{-3}\right)$-one obtains

$$
\begin{aligned}
{[\hat{H}, \hat{F}]=} & \frac{1}{2 m}\left[p^{2}, F\right]-\frac{1}{8 m^{3} c^{2}}\left[p^{4}, F\right]+\frac{\hbar^{2}}{16 m^{3} c^{2}}\left[p^{2}, \vec{\nabla} \cdot \vec{G}\right] \\
& +\frac{\hbar}{8 m^{3} c^{2}}\left[p^{2}, \vec{\sigma} \cdot \vec{G} \times \vec{p}\right]+\frac{\hbar e}{4 m^{2} c^{2}}[V(\vec{r}), \vec{\sigma} \cdot \vec{G} \times \vec{p}] \\
& +\frac{\hbar e}{4 m^{2} c^{2}}[F, \vec{\sigma} \cdot \vec{E} \times \vec{p}] .
\end{aligned}
$$

The Hermitian conjugate of $\hat{F}$ can be obtained from Eq. (5) in the following form:

$$
\begin{aligned}
\hat{F}^{\dagger}(\vec{r})= & F^{\dagger}(\vec{r})+\frac{\hbar^{2}}{8 m^{2} c^{2}} \vec{\nabla} \cdot \vec{G}^{\dagger}-\frac{\hbar}{4 m^{2} c^{2}} \vec{\sigma} \cdot \vec{p} \times \vec{G}^{\dagger} \\
& +\frac{\hbar^{2} e}{4 m^{3} c^{4}}\left(\vec{E} \cdot \vec{G}^{\dagger}\right) .
\end{aligned}
$$

Hence, to $O\left(\mathrm{~m}^{-3}\right)$, one obtains from Eqs. (6) and (7) the following result:

$$
\begin{aligned}
\hat{F}^{\dagger}[\hat{H}, \hat{F}]= & \frac{1}{2 m} F^{\dagger}\left[p^{2}, F\right]-\frac{1}{8 m^{3} c^{2}} F^{\dagger}\left[p^{4}, F\right] \\
& +\frac{\hbar^{2}}{16 m^{3} c^{2}}\left\{F^{\dagger}\left[p^{2}, \vec{\nabla} \cdot \vec{G}\right]+\left(\vec{\nabla} \cdot \vec{G}^{\dagger}\right)\left[p^{2}, F\right]\right\} \\
& +\frac{\hbar}{8 m^{3} c^{2}}\left\{F^{\dagger}\left[p^{2}, \vec{\sigma} \cdot \vec{G} \times \vec{p}\right]+\vec{\sigma} \cdot \vec{p} \times \vec{G}^{\dagger}\left[F, p^{2}\right]\right\} \\
& +\frac{\hbar e}{4 m^{2} c^{2}}\left\{F^{\dagger}[V(\vec{r}), \vec{\sigma} \cdot \vec{G} \times \vec{p}]+F^{\dagger}[F, \vec{\sigma} \cdot \vec{E} \times \vec{p}]\right\} .
\end{aligned}
$$

Substitution of Eq. (8) into Eq. (2a) gives the lowest relativistic corrections to the generalized sum rule. The first term in Eq. (8) leads back to the nonrelativistic result (except for possible corrections from the eigenstates as discussed below). The last two terms in Eq. (8) involving the spin $\vec{\sigma}$ will not contribute to the sum since they all contain odd powers of the momentum operator [7]. Thus the relativistic corrections mainly come from the second and third terms of Eq. (8). Hence, using the first three terms in Eq. (8) and with $\vec{p}=-i \hbar \vec{\nabla}$, we finally obtain the following result for the sum rule with relativistic corrections to the lowest order:

$$
\begin{aligned}
S= & \sum_{n}\left(E_{n}-E_{0}\right)|\langle n|F(\vec{r})| 0\rangle|^{2} \\
= & -\frac{\hbar^{2}}{2 m}\left\langle 0\left|F^{\dagger} \nabla^{2} F+2 F^{\dagger} \vec{\nabla} F \cdot \vec{\nabla}\right| 0\right\rangle-\frac{\hbar^{4}}{8 m^{3} c^{2}}\langle 0| F^{\dagger} \nabla^{4} F \\
& +2 F^{\dagger} \nabla^{2} F \nabla^{2}+4 F^{\dagger}\left(\partial_{j} \partial_{k} F\right)\left(\partial_{j} \partial_{k}\right)+4 F^{\dagger} \vec{\nabla}\left(\nabla^{2} F\right) \cdot \vec{\nabla} \\
& +4 F^{\dagger} \vec{\nabla} F \cdot \vec{\nabla} \nabla^{2}|0\rangle-\frac{\hbar^{4}}{16 m^{3} c^{2}}\langle 0| F^{\dagger} \nabla^{4} F+\nabla^{2} F^{\dagger} \nabla^{2} F \\
& +2 F^{\dagger} \vec{\nabla}\left(\nabla^{2} F\right) \cdot \vec{\nabla}+2\left(\nabla^{2} F^{\dagger}\right) \vec{\nabla} F \cdot \vec{\nabla}|0\rangle .
\end{aligned}
$$

Note that the summation convention of repeated indices has been assumed in Eq. (9), which constitutes our main result in the present work. Note also that the eigenstates in Eq. (9) refer to the normalized positive energy states of the Hamiltonian in Eq. (4) which form a complete set. The ground eigenstate of these was first obtained by Berestetskii and Landau [15] in the following form:

$$
|0\rangle=\left(1-\frac{\hbar^{2}}{8 m^{2} c^{2}} \nabla^{2}\right)|0\rangle_{D}
$$

where $|0\rangle_{D}$ is the large component of the exact Dirac eigenstate which can in turn be expressed in terms of the nonrelativistic Schrödinger state as [16]

$$
\langle r \mid 0\rangle_{D}=N^{\prime} r^{\gamma-1}\langle r \mid 0\rangle_{S} \chi_{ \pm}=N^{\prime} r^{\gamma-1} e^{-Z r / a} \chi_{ \pm} .
$$

In Eq. (11), $N^{\prime}$ is an appropriate normalization constant, $a$ the Bohr radius, $\gamma=\sqrt{1-Z^{2} \alpha^{2}}$ with $\alpha$ the fine structure constant, and $\chi_{ \pm}$the up-down two-component Pauli spinors. Note that to the lowest-order correction in the sum $S$, corrections from the eigenstate $|0\rangle$ will at most enter via the first term in Eq. (9), which simply resembles the nonrelativistic sum had the eigenstate $|0\rangle$ assumed the Schrödinger state. The second and third terms in Eq. (9) represent relativistic corrections originating from the Hamiltonian and from the transformed $F(\vec{r})$, respectively, which can be combined into one term in principle. Note that corrections from the eigenstate $|0\rangle$ will not enter into these two terms since these will lead to higher-order terms. This is in complete analogy to ordinary perturbation theory in which zeroth-order eigenfunctions are used to calculate first-order corrections to eigen energies. Hence, to lowest-order corrections, one simply uses the Schrödinger states for $|0\rangle$ in the second and third terms in Eq. (9). Note also that the result in Eq. (9) can in principle be symmetrized using Eq. (2b) as in the result obtained for the nonrelativistic case in Eq. (2c). Since the result will be very involved in the relativistic case, we shall leave it in the unsymmetrized form as in Eq. (9). However, the symmetrization process will still be very useful to obtain simplification in actual calculations as shown below. 
As in Wang [9] for the nonrelativistic case, we shall illustrate the application of Eq. (9) to obtain relativistic corrections to the following three sum rules.

(i) The Bethe sum rule. For $F=e^{i \vec{q} \cdot \vec{r}}$, where $\vec{q}$ is the momentum transfer which is a $c$ number, we obtain from Eq. (9) the following result for the Bethe sum rule:

$$
\begin{aligned}
S_{B}= & \frac{\hbar^{2}}{2 m}\left\langle 0\left|q^{2}-2 i \vec{q} \cdot \vec{\nabla}\right| 0\right\rangle-\frac{\hbar^{4}}{8 m^{3} c^{2}}\langle 0| q^{4}-2 q^{2} \nabla^{2} \\
& -4(\vec{q} \cdot \vec{\nabla})^{2}-4 i q^{2} \vec{q} \cdot \vec{\nabla}+4 i \vec{q} \cdot \vec{\nabla} \nabla^{2}|0\rangle \\
& -\frac{\hbar^{4}}{16 m^{3} c^{2}}\left\langle 0\left|2 q^{4}-4 i q^{2} \vec{q} \cdot \vec{\nabla}\right| 0\right\rangle .
\end{aligned}
$$

Since it can be shown that all the matrix elements with an odd power in the gradient operator will vanish [7], we obtain from Eq. (12) the Bethe sum rule up to the lowest order in relativistic corrections in the following form:

$$
S_{B}=\frac{\hbar^{2} q^{2}}{2 m}+\Delta S_{B},
$$

where

$\Delta S_{B}=-\frac{\hbar^{4} q^{4}}{4 m^{3} c^{2}}+\frac{\hbar^{4} q^{2}}{4 m^{3} c^{2}}\left\langle 0\left|\nabla^{2}\right| 0\right\rangle+\frac{\hbar^{4}}{2 m^{3} c^{2}}\left\langle 0\left|(\vec{q} \cdot \vec{\nabla})^{2}\right| 0\right\rangle$.

The result in Eq. (14) reproduces a result obtained previously in the literature [3]. Note that the corrections from the eigenstate do not enter the calculation of this sum since $q^{2}$ in the leading term is a constant, and the state $|0\rangle$ appearing in Eq. (14) is simply the nonrelativistic Schrödinger eigenstate.

(ii) The TRK sum rule. Although the TRK sum rule can in principle be obtained as the low- $\vec{q}$ limit of the Bethe sum rule $[3,11]$, here we demonstrate explicitly how the result in Eq. (9) can be applied directly to obtain relativistic corrections to this sum rule. For $\vec{F}=\vec{r}$, which is a vector operator, one simply needs to replace $F$ in Eq. (9) by a component of $\vec{F}$ and sum over the contributions from each of the components [9]. Since here the function is linear in the coordinates, only the first-order derivatives will survive and we find from Eq. (9) the following:

$S_{T R K}=-\frac{\hbar^{2}}{2 m}\left\langle 0\left|2 x_{i} \vec{\nabla} x_{i} \cdot \vec{\nabla}\right| 0\right\rangle-\frac{\hbar^{4}}{8 m^{3} c^{2}}\left\langle 0\left|4 x_{i} \vec{\nabla} x_{i} \cdot \vec{\nabla} \nabla^{2}\right| 0\right\rangle$,

where summation over the index $i$ is implied. The evaluation of the expectation values in Eq. (15) can be facilitated by using the symmetrized form (2b) which is equivalent to the "double-commutator" calculation for Hermitian $F$. When this is done, one obtains the following results for the integrals of the expectation values:

$$
\begin{gathered}
I_{1}=\left\langle 0\left|x_{i} \vec{\nabla} x_{i} \cdot \vec{\nabla}\right| 0\right\rangle=-\frac{3}{2}, \\
I_{2}=\left\langle 0\left|x_{i} \vec{\nabla} x_{i} \cdot \vec{\nabla} \nabla^{2}\right| 0\right\rangle=-\frac{5}{2}\left\langle 0\left|\nabla^{2}\right| 0\right\rangle .
\end{gathered}
$$

Substitution of the results in Eqs. (16) and (17) into Eq. (15) yields the TRK sum rule to the lowest-order relativistic corrections in the following form:

$$
S_{T R K}=\frac{3 \hbar^{2}}{2 m}+\frac{5 \hbar^{4}}{4 m^{3} c^{2}}\left\langle 0\left|\nabla^{2}\right| 0\right\rangle .
$$

We see that the result in Eq. (18) again reproduces the previous result obtained in the literature [3], with $|0\rangle$ being simply the Schrödinger state.

(iii) The monopole sum rule. For $F=r^{2}$, Wang has used the generalized result in Eq. (1) to obtain the nonrelativistic result for the monopole sum rule which has its application in nuclear collective excitation [9,17]. Using Eq. (9), we obtain the nonvanishing terms for the monopole sum rule to the lowest-order relativistic corrections as follows:

$$
\begin{aligned}
S_{\text {mono }}= & -\frac{\hbar^{2}}{2 m}\left\langle 0\left|r^{2} \nabla^{2} r^{2}+2 r^{2} \vec{\nabla} r^{2} \cdot \vec{\nabla}\right| 0\right\rangle-\frac{\hbar^{4}}{8 m^{3} c^{2}}\langle 0| 2 r^{2} \nabla^{2} r^{2} \nabla^{2} \\
& +4 r^{2}\left(\partial_{j} \partial_{k} r^{2}\right)\left(\partial_{j} \partial_{k}\right)+4 r^{2} \vec{\nabla} r^{2} \cdot \vec{\nabla} \nabla^{2}|0\rangle \\
& -\frac{\hbar^{4}}{16 m^{3} c^{2}}\left\langle 0\left|\nabla^{2} r^{2} \nabla^{2} r^{2}+2\left(\nabla^{2} r^{2}\right) \vec{\nabla} r^{2} \cdot \vec{\nabla}\right| 0\right\rangle .
\end{aligned}
$$

To evaluate the expectation values, we again resort to the symmetrization approach and obtain for the nonrelativistic term:

$$
I_{3}=\left\langle 0\left|r^{2} \nabla^{2} r^{2}+2 r^{2} \vec{\nabla} r^{2} \cdot \vec{\nabla}\right| 0\right\rangle=-4\left\langle 0\left|r^{2}\right| 0\right\rangle .
$$

For the relativistic correction, we have the following expectation values:

$$
\begin{aligned}
I_{4} & =\left\langle 0\left|2 r^{2} \nabla^{2} r^{2} \nabla^{2}+4 r^{2}\left(\partial_{j} \partial_{k} r^{2}\right)\left(\partial_{j} \partial_{k}\right)+4 r^{2} \vec{\nabla} r^{2} \cdot \vec{\nabla} \nabla^{2}\right| 0\right\rangle \\
& =-60-80\left\langle 0\left|r \frac{\partial}{\partial r}\right| 0\right\rangle-16\left\langle 0\left|r^{2} \frac{\partial^{2}}{\partial r^{2}}\right| 0\right\rangle-8\left\langle 0\left|r^{2} \nabla^{2}\right| 0\right\rangle
\end{aligned}
$$

and

$$
I_{5}=\left\langle 0\left|\nabla^{2} r^{2} \nabla^{2} r^{2}+2\left(\nabla^{2} r^{2}\right) \vec{\nabla} r^{2} \cdot \vec{\nabla}\right| 0\right\rangle=36+24\left\langle 0\left|r \frac{\partial}{\partial r}\right| 0\right\rangle \text {, }
$$

where we have used the following results: $\nabla^{2} r^{2}=6, \vec{\nabla} r^{2}$ $=2 r \hat{r}, \ldots$, etc. Substituting Eqs. (20)-(22) into Eq. (19), we finally obtain the monopole sum rule to the lowest-order relativistic correction in the following form:

$$
S_{\text {mono }}=\frac{2 \hbar^{2}}{m}\left\langle 0\left|r^{2}\right| 0\right\rangle+\Delta S_{\text {mono }}^{\prime},
$$

where for spherical symmetric ground state we have

$$
\Delta S_{\text {mono }}^{\prime}=\frac{3 \hbar^{4}}{4 m^{3} c^{2}}\left(7+14\left\langle 0\left|r \frac{\partial}{\partial r}\right| 0\right\rangle+4\left\langle 0\left|r^{2} \frac{\partial^{2}}{\partial r^{2}}\right| 0\right\rangle\right) \text {. }
$$

Note that in this case, there will be relativistic contributions to the first term in Eq. (23) through the eigenstate $|0\rangle$ given in Eq. (10), while that appearing in Eq. (24) is still simply the Schrödinger state. Explicit calculation of all these corrections in Eqs. (14), (18), (23), and (24) using the appropriate eigenstates is straightforward, but can be tedious, and is given in the Appendix.

In this work, we have derived the lowest-order relativistic corrections to a generalized sum rule for an arbitrary function of the coordinates which does not need to be Hermitian. 
When corrections from the eigenstates cannot be ignored, the calculation can be quite tedious as illustrated in the Appendix. Other possible applications of our present result in Eq. (9) include corrections to various sum rules useful for nuclear systems where $F(\vec{r})$ assumes the density operator of the nucleons [1]. In principle, as long as the transition probability (or form factor) depends only on the spatial coordinates of the system involved, our result will be useful for the study of the relativistic corrections to the calculations of total cross sections, stopping powers, etc., as has been illustrated previously in the literature [8].

H.S. acknowledges the support of the government of Saudi Arabia, and P.T.L. acknowledges useful discussions with Professor G. J. Ni.

\section{APPENDIX}

Here we give the explicit results for the lowest-order correction to the three sums discussed in the text. For the Bethe and TRK sum rules, using the Schrödinger hydrogenic ground state in the results in Eqs. (14) and (18), one easily obtains the following explicit results $[7,8]$ :

$$
\begin{gathered}
\Delta S_{B}=-\frac{\hbar^{4}}{8 m^{3} c^{2}}\left(q^{4}+\frac{10}{3} \frac{q^{2} Z^{2}}{a^{2}}\right), \\
\Delta S_{T R K} \equiv \frac{5 \hbar^{4}}{4 m^{3} c^{2}}\left\langle 0\left|\nabla^{2}\right| 0\right\rangle=-\frac{5 \hbar^{4}}{4 m^{3} c^{2}} \frac{Z^{2}}{a^{2}} .
\end{gathered}
$$

To obtain consistently the corrections to the monopole sum rule, one must apply the eigenstates given in Eqs. (10) and (11) to the leading term of Eq. (23). The calculation of Eq. (24) using nonrelativistic $|0\rangle$ is rather simple and leads to

$$
\Delta S_{\text {mono }}^{\prime}=-\frac{3}{2} \frac{\hbar^{4}}{m^{3} c^{2}} \text {. }
$$

To extract the relativistic effects from using the state in Eq. (10) to calculate

$$
S_{\text {mono }}^{(0)} \equiv \frac{2 \hbar^{2}}{m}\left\langle 0\left|r^{2}\right| 0\right\rangle
$$

we first substitute Eq. (11) into Eq. (10) and obtain to lowest order the normalized semirelativistic wave function for the ground state. Using this ground state in Eq. (A4) and after some tedious algebra, one finally obtains the following result:

$$
\begin{aligned}
S_{\text {mono }}^{(0)}= & \frac{\hbar^{2}}{2 m}\left(\frac{a}{Z}\right)^{2} \frac{\Gamma(2 \gamma+3)}{\Gamma(2 \gamma+1)}-\frac{\hbar^{4}}{2 m^{3} c^{2}} \frac{\Gamma(2 \gamma+3)}{\Gamma(2 \gamma+1)} \\
& \times\left[\frac{\Gamma(2 \gamma)}{\Gamma(2 \gamma+1)}-\frac{\Gamma(2 \gamma+2)}{\Gamma(2 \gamma+3)}\right] .
\end{aligned}
$$

Recalling that $\gamma=\sqrt{1-(Z \alpha)^{2}} \approx 1-\frac{1}{2} Z^{2} \alpha^{2}$ to the lowest order of the fine structure constant, the result in Eq. (A5) can finally be simplified to include up to the lowest-order relativistic correction terms in the following form:

$$
S_{\text {mono }}^{(0)}=\frac{6 \hbar^{2}}{m}\left(\frac{a}{Z}\right)^{2}-\frac{3 \hbar^{4}}{2 m^{3} c^{2}}\{1-4[\psi(3)-\psi(5)]\},
$$

where the first term in Eq. (A6) is the nonrelativistic result and $\psi(x)$ in the second term is defined as the logarithmic derivative of the gamma function. Thus, combining the results in Eqs. (A3) and (A6) and using the following expansion for $\psi(n)$ [18],

$$
\psi(n+1)=-\gamma+\sum_{k=1}^{n} \frac{1}{k}
$$

where $\gamma$ is Euler's constant, we finally obtain the total correction term as follows:

$$
\Delta S_{\text {mono }}=-\frac{13}{2} \frac{\hbar^{4}}{m^{3} c^{2}} .
$$

[1] For an earlier comprehensive review, see, e.g., G. Orlandini and M. Traini, Rep. Prog. Phys. 54, 257 (1991); see also R. Jackiw, Phys. Rev. 157, 1220 (1967).

[2] S. P. Goldman and G. W. F. Drake, Phys. Rev. A 25, 2877 (1982).

[3] See, e.g., S. M. Cohen and P. T. Leung, Phys. Rev. A 57, 4994 (1998); and references therein.

[4] K.-M. Schmitt and H. Arenhovel, Z. Phys. A 320, 311 (1985).

[5] G. A. Aucar et al., Phys. Rev. A 52, 1054 (1995).

[6] See S. M. Cohen, Adv. Quantum Chem. 46, 241 (2004).

[7] See also, P. T. Leung et al., Phys. Rev. A 33, 2827 (1986).

[8] P. T. Leung, Phys. Rev. A 40, 5417 (1989).

[9] S. Wang, Phys. Rev. A 60, 262 (1999).

[10] Note that Wang has actually considered a sum of the form $\Sigma_{n}\left(E_{n}-E_{0}\right)|\langle 0|F(\vec{r})| n\rangle|^{2}$ which is different from Eq. (1) if $F(\vec{r})$ is not Hermitian. Here we shall consider Eq. (1) as the more standard one which finds applications in many physical processes. In addition, the "double-commutator" calculation in [9] is valid only for Hermitian $F(\vec{r})$.
[11] H. A. Bethe and R. W. Jackiw, Intermediate Quantum Mechanics (Addison-Wesley Longman, Boulder, CO, 1997).

[12] In passing, we also note that since $F=e^{i \vec{q} \cdot \vec{r}}$ is unitary rather than Hermitian, Wang's definition of the sum (see the footnote [10] above) indeed yields formally a different result which has a positive sign in the second term of the RHS of Eq. (3). However, since this term vanishes anyway, Wang's result also reproduces the Bethe sum rule in this particular case.

[13] D. Y. Smith, Phys. Rev. A 35, 3381 (1987).

[14] H. Sinky, Ph.D. thesis, Portland State University, 2005 (unpublished).

[15] V. B. Beresteskii et al., Quantum Electrodynamics (Pergamon, New York, 1982), p. 125.

[16] See, e.g., J. D. Bjorken and S. D. Drell, Relativistic Quantum Mechanics (McGraw-Hill, New York, 1964).

[17] Note that the result presented in Eq. (20) of Ref. [9] is off by a factor of 4.

[18] See, e.g., L. C. Andrews, Special Functions of Mathematics for Engineers, 2nd ed. (Oxford University Press, Oxford, 1998). 